\title{
LXXVI. Meteorological observations during a residence in colombia between the years 1820 and 1830
}

\section{Colonel Richard Wright}

To cite this article: Colonel Richard Wright (1839) LXXVI. Meteorological observations during a residence in colombia between the years 1820 and 1830, Philosophical Magazine Series 3, 15:99, 508-517, DOI: $10.1080 / 14786443908649948$

To link to this article: http://dx.doi.org/10.1080/14786443908649948

曲 Published online: 01 Jun 2009.

Submit your article to this journal $[\pi$

Џll Article views: 2

Q View related articles $₫$ 


\section{[ 508 ]}

LXXVI. Meteorological Observations during a Residence in Colombia betreen the Years 1820 and 1830. By Colonel Richand Wright, Governor of the Province of Loxa, Confidential Agent of the Republic of the Equator, \&c. \&c.

[Continued from p. 380, and concluded.]

Sanmillan; suburbs of Quito, on the plain of Anaquito, about half a mile north of the City. Situation open.

\begin{tabular}{|c|c|c|c|c|c|}
\hline Date. & $\begin{array}{l}\text { Thermo- } \\
\text { meter. }\end{array}$ & Time. & $\begin{array}{l}\text { Hygro- } \\
\text { meter. }\end{array}$ & Time. & Remarks. \\
\hline $\begin{array}{r}1827 . \\
\text { May } 26 . \\
27 . \\
28 . \\
\text { "̈. } \\
29 . \\
3 \ddot{0} . \\
3 \ddot{31} \\
\text { ". }\end{array}$ & $\begin{array}{c}\circ \\
", \\
45 \cdot 0 \\
61 \cdot 0 \\
43 \cdot 0 \\
63 \cdot 0 \\
56 \cdot 0 \\
64 \cdot 0 \\
52 \cdot 0 \\
66 \cdot 0\end{array}$ & 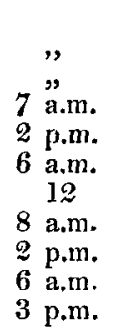 & $\begin{array}{l}27 \cdot 5 \\
22 \cdot 0 \\
27 \cdot 5\end{array}$ & $\begin{array}{c}12 \\
2 \text { p.m. } \\
3 \text { p.m. }\end{array}$ & $\begin{array}{l}\text { Rain. } \\
\text { Clondy. } \\
\text { Fair. } \\
\text { Showery. } \\
\text { Fair. } \\
\text { Id. }\end{array}$ \\
\hline \multicolumn{6}{|c|}{4 days. $\left.\begin{array}{l}62^{\circ} .5 \max . \\
46^{\circ} .66 \min .\end{array}\right\} 540.58$ med. } \\
\hline 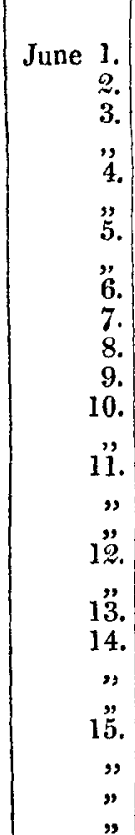 & $\begin{array}{l}53 \cdot 0 \\
63 \cdot 0 \\
5 \cdot 0 \\
63 \cdot 0 \\
48 \cdot 0 \\
65 \cdot 5 \\
48 \cdot 0 \\
66 \cdot 0 \\
52 \cdot 0 \\
65 \cdot 0 \\
63 \cdot 0 \\
64 \cdot 0 \\
53 \cdot 0 \\
65 \cdot 0 \\
43 \cdot 0 \\
54 \cdot 0 \\
65 \cdot 5 \\
52 \cdot 0 \\
65 \cdot 0 \\
67 \cdot 0 \\
51 \cdot 0 \\
76 \cdot 0 \\
64 \cdot 0 \\
52 \cdot 0 \\
57 \cdot 0 \\
59 \cdot 5 \\
60 \cdot 0\end{array}$ & 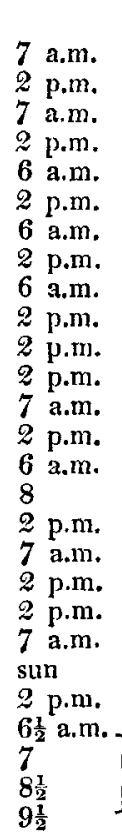 & 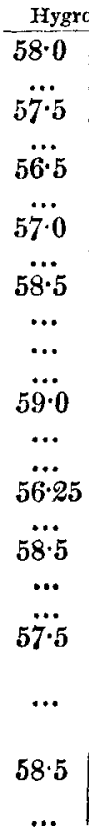 & $\begin{array}{l}38 \cdot 5 \\
\\
38 \cdot 5 \\
16 \cdot 5 \\
55 \cdot 0 \\
55 \cdot 0\end{array}$ & $\begin{array}{l}\text { Cloudy ; rain at night. } \\
\text { Cloudy. } \\
\text { Fair. } \\
\text { Showery. } \\
\text { Id. } \\
\text { Id. } \\
\text { Fair. } \\
\text { Id. } \\
\text { Id. } \\
\text { Id. } \\
\text { Cloudy. }\end{array}$ \\
\hline
\end{tabular}


Col.Wright's Metecrological Observations in Colombia. 509

Sanmillan-TABrs continued.

\begin{tabular}{|c|c|c|c|c|c|}
\hline Date. & $\begin{array}{l}\text { Thermo- } \\
\text { meter. }\end{array}$ & Time. & Hygro & neter. & Remarks. \\
\hline 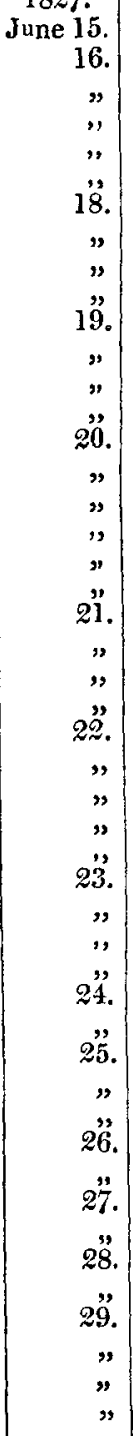 & $\begin{array}{l}64 \cdot 0 \\
55 \cdot 0 \\
58 \cdot 0 \\
62 \cdot 0 \\
66 \cdot 0 \\
80 \cdot 0 \\
48 \cdot 0 \\
61 \cdot 0 \\
53 \cdot 0 \\
63 \cdot 5 \\
49 \cdot 0 \\
55 \cdot 5 \\
75 \cdot 0 \\
67 \cdot 0 \\
48 \cdot 0 \\
61 \cdot 0 \\
58 \cdot 0 \\
74 \cdot 0 \\
64 \cdot 0 \\
82 \cdot 0 \\
45 \cdot 0 \\
55 \cdot 0 \\
74 \cdot 0 \\
64 \cdot 0 \\
44 \cdot 0 \\
52 \cdot 0 \\
59 \cdot 0 \\
66 \cdot 0 \\
82 \cdot 0 \\
54 \cdot 0 \\
71 \cdot 0 \\
64 \cdot 0 \\
80 \cdot 0 \\
53 \cdot 0 \\
64 \cdot 0 \\
49 \cdot 0 \\
56 \cdot 0 \\
75 \cdot 0 \\
51 \cdot 5 \\
66 \cdot 0 \\
53 \cdot 0 \\
67 \cdot 0 \\
55 \cdot 0 \\
64 \cdot 0 \\
50 \cdot 0 \\
60 \cdot 0 \\
64 \cdot 0 \\
69 \cdot 0\end{array}$ & 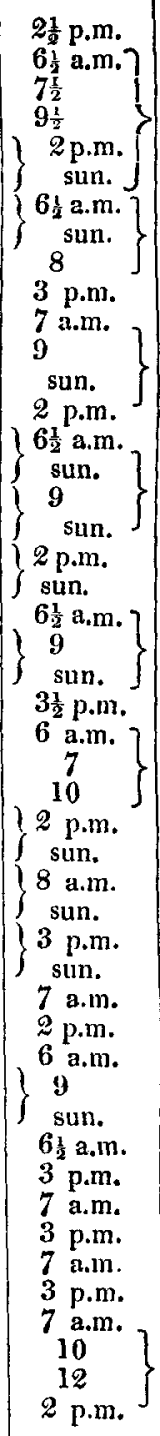 & $\begin{array}{c}\ldots \\
59 \cdot 0 \\
\ldots \\
58 \cdot 5 \\
\ldots \\
52 \cdot 5 \\
58 \cdot 75 \\
\ldots \ddot{0} \cdot 0 \\
\ldots \ddot{9} \cdot 5 \\
\cdots \\
59 \cdot 0\end{array}$ & $\begin{array}{r}55 \cdot 0 \\
66.0 \\
55.0\end{array}$ & $\begin{array}{l}\text { Id. } \\
\text { Clear and strong winds } \\
\text { from N.E. } \\
\text { Cloudy and showery. }\end{array}$ \\
\hline
\end{tabular}




\section{Col. R. Wright's Meteorological Observations made}

Sanmillan.-TABLE continued.

\begin{tabular}{|c|c|c|c|c|c|}
\hline Date. & $\begin{array}{l}\text { Thermo- } \\
\text { meter. }\end{array}$ & Time. & \multicolumn{2}{|c|}{ Fygrometer. } & Remarks. \\
\hline $\begin{array}{r}1827 . \\
\text { June } 30 . \\
\text { " } \\
\text { " } \\
\text { " }\end{array}$ & $\left.\begin{array}{l}48 \cdot 0 \\
54 \cdot 5 \\
56 \cdot 0 \\
74 \cdot 0 \\
65 \cdot 0 \\
81 \cdot 0\end{array}\right\}$ & 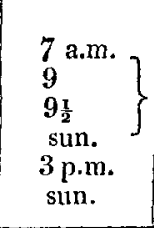 & $\cdots$ & $22 \cdot 0$ & $\begin{array}{l}\text { Cloudy and damp. } \\
\text { Fair. }\end{array}$ \\
\hline 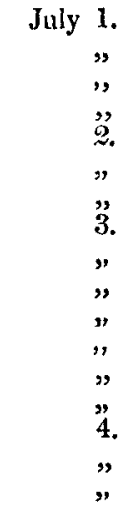 & $\left.\begin{array}{l}49 \cdot 0 \\
59 \cdot 0 \\
63 \cdot 0 \\
60 \cdot 0 \\
53 \cdot 0 \\
65 \cdot 0 \\
60 \cdot 0 \\
54 \cdot 0 \\
64 \cdot 0 \\
62 \cdot 0 \\
74 \cdot 0 \\
66 \cdot 0 \\
84 \cdot 0 \\
53 \cdot 0 \\
53 \cdot 0 \\
65 \cdot 0 \\
53 \cdot 0\end{array}\right\}$ & 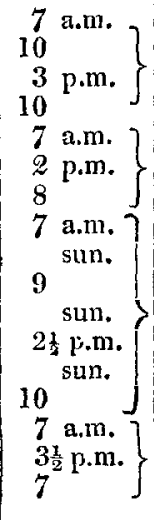 & $\ddot{5} \ddot{2} \cdot 25$ & $66 \cdot 0$ & $\begin{array}{l}\text { Clear. } \\
\text { Id. and wind. }\end{array}$ \\
\hline \multicolumn{6}{|c|}{34 days. - Thermomer mean $57^{\circ .25}$. -Hygrometer $47^{\circ} \cdot 75$. Var. $60^{\circ} \cdot 5$. } \\
\hline $\begin{array}{l}\text { August } \\
31 \text { days. }\end{array}$ & \multicolumn{5}{|c|}{$\left.\begin{array}{l}67^{\circ} .37 \text { max. } \\
52 \cdot 5 \text { min. } \\
\text { Weather generally fair and windy. }\end{array}\right\}$} \\
\hline $\begin{array}{l}\text { Septemb. } \\
30 \text { days. }\end{array}$ & \multicolumn{5}{|c|}{ 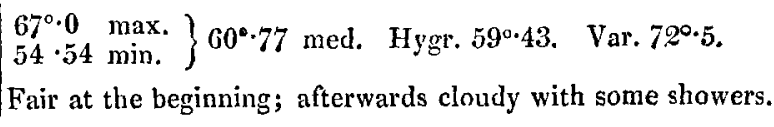 } \\
\hline $\begin{array}{l}\text { October } \\
12 \text { days. }\end{array}$ & $61^{0 \cdot 7}$ & $\max .\} 57^{\circ} \cdot 5$ & 5 med. & Hyg & $28^{\circ} \cdot 15$. Var. $27^{\circ} \cdot 5$ \\
\hline $\begin{aligned} \text { Dec. } & 1 . \\
& \\
& \\
& \\
& \\
& \\
& 7 . \\
& \\
& \end{aligned}$ & $\begin{array}{l}51 \cdot 0 \\
72 \cdot 5 \\
51 \cdot 0 \\
74 \cdot 0 \\
61 \cdot 0 \\
51 \cdot 0 \\
71 \cdot 0 \\
64 \cdot 0\end{array}$ & $\left.\begin{array}{l}6 \text { a.m. } \\
2 \text { p.m. } \\
6 \text { a.m. } \\
2 \text { p.m. } \\
5 \\
6 \text { a.m. } \\
\left.\begin{array}{r}2 \frac{1}{2} \\
5\end{array}\right\} . m .\end{array}\right\}$ & $\begin{array}{l}61 \cdot 75 \\
62 \cdot 0 \\
62 \cdot 0\end{array}$ & & $\begin{array}{l}\text { Mornings clear : evenings } \\
\text { cloudy. }\end{array}$ \\
\hline
\end{tabular}


Sanmillan.-TABLE continued.

\begin{tabular}{|c|c|c|c|c|}
\hline Date. & $\begin{array}{l}\text { Thermo. } \\
\text { meter. }\end{array}$ & Time. & Hygrometer. & Remarks. \\
\hline 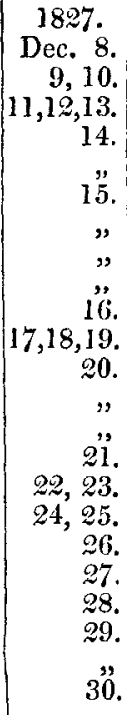 & $\begin{array}{l}7 \stackrel{0}{2} \cdot 0 \\
70 \cdot 0 \\
72 \cdot 0 \\
74 \cdot 0 \\
127 \cdot 0 \\
52 \cdot 0 \\
73 \cdot 0 \\
60 \cdot 0 \\
56 \cdot 0 \\
69 \cdot 0 \\
68 \cdot 0 \\
51 \cdot 0 \\
55 \cdot 0 \\
70 \cdot 0 \\
79 \cdot 0 \\
72 \cdot 0 \\
74 \cdot 0 \\
71 \cdot 0 \\
72 \cdot 0 \\
69 \cdot 0 \\
51 \cdot 0 \\
71 \cdot 0 \\
60 \cdot 0\end{array}$ & 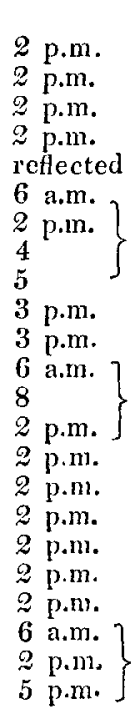 & $58 \cdot 66$ & $\begin{array}{l}\text { Storm. } \\
\text { Id. } \\
\text { Fair. } \\
\text { Id. } \\
9 \text { p.m. shock of an earth- } \\
\text { Fair. } \\
\text { Id. } \\
\text { Id. } \\
\text { Id. } \\
\text { Rain. }\end{array}$ \\
\hline \multicolumn{5}{|c|}{$\begin{array}{l}25 \text { days. } 60^{\circ} \cdot 88 \text { mean. Var. } 22^{\circ} . \text { Maximum of heat } 74^{\circ} \cdot 0: \text { extraordinary } \\
\text { in Quito. Vide Humboldt, De Geog. Plant.,p. } 103 \text {. }\end{array}$} \\
\hline
\end{tabular}

Comparative Temperature of different Parts of the Table-land of the Equator.

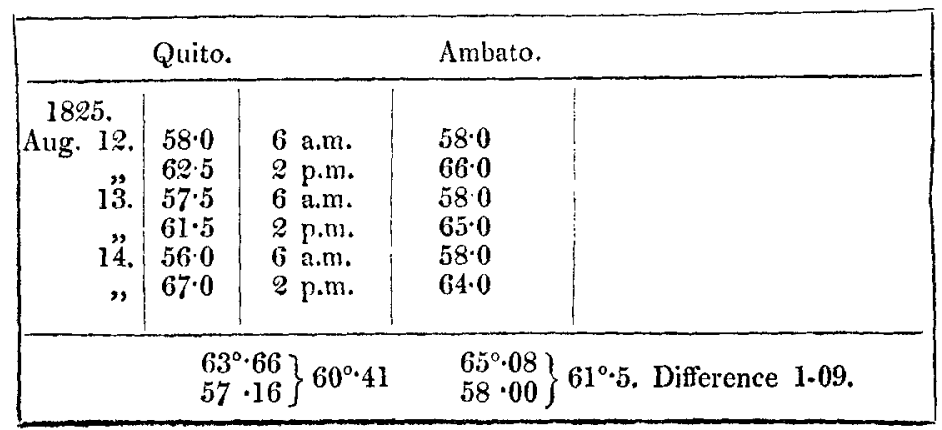


512 Col. R. Wright's Meteorological Observations made

Comp. Temp.-TaвLE continued.

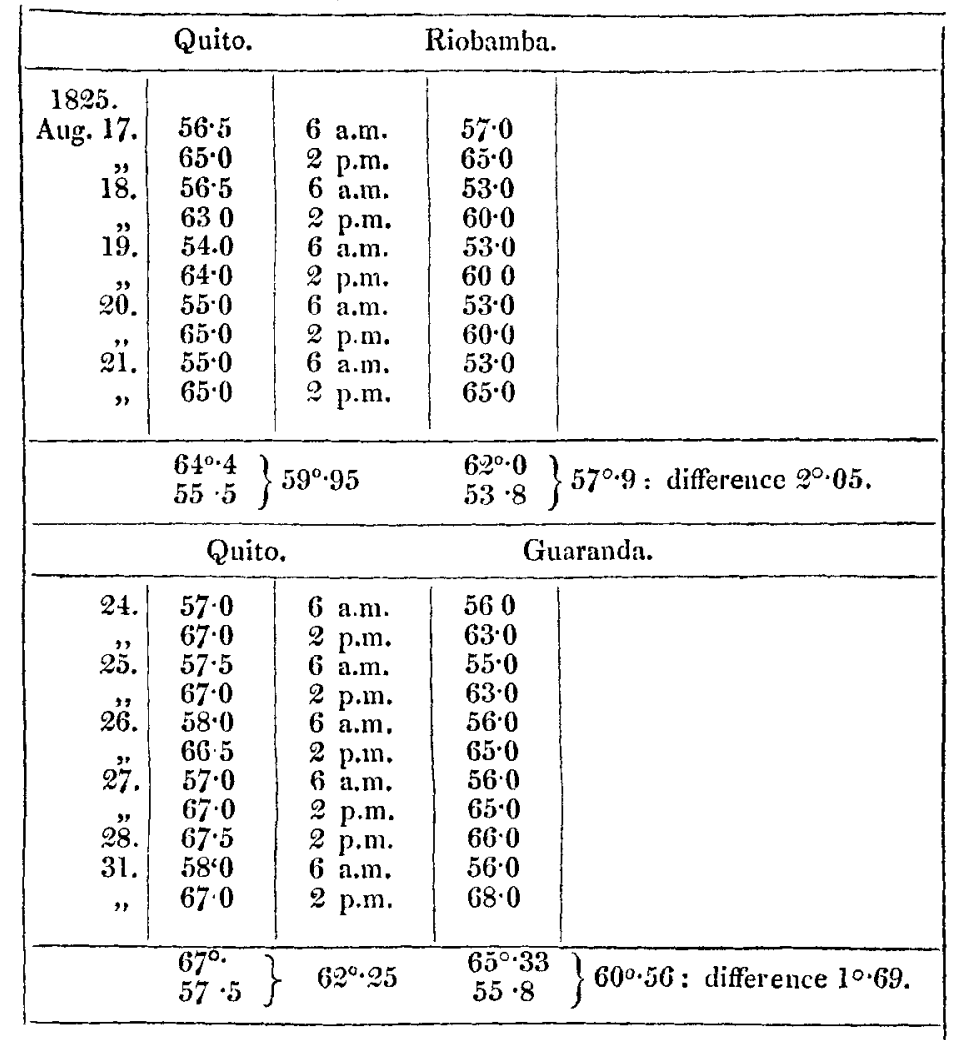

Village of Cayambe. Lat. 1'35"N. Elevation 9724, feet. B. W. $195^{\circ} \frac{9}{10}$.

\begin{tabular}{|c|c|c|c|c|}
\hline Date. & $\begin{array}{l}\text { Thermo- } \\
\text { meter. }\end{array}$ & Time. & Iygrometer. & Remarks. \\
\hline $\begin{array}{rr}1827 . \\
\text { July } \\
8 . \\
9 \\
9 . \\
10 . \\
1 " \\
11 . \\
12 . \\
"\end{array}$ & $\left.\begin{array}{l}52 \cdot 0 \\
64 \cdot 0 \\
52 \cdot 0 \\
57 \cdot 0 \\
59 \cdot 0 \\
60 \cdot 0 \\
64 \cdot 0 \\
56 \cdot 5 \\
71 \cdot 0 \\
64 \cdot 0\end{array}\right\}$ & $\begin{aligned} & 7 \text { a.m. } \\
& 3 \text { p.m. } \\
& 7 \text { a.m. } \\
& 7 \text { a.m. } \\
& 10 \\
& 4 \text { p.m. } \\
& 2 \text { p.m. } \\
& 8 \text { a.m. } \\
& \text { sum. } \\
& 3 \text { p.m. }\end{aligned}$ & $\begin{array}{l}66^{\circ} 0 \\
47 \cdot 5 \\
75 \cdot 0 \\
75 \cdot 0\end{array}$ & $\begin{array}{l}\text { Fair and clear. } \\
\text { ld. } \\
\text { Cloudy. } \\
\text { Fair and windy. } \\
\text { Id. }\end{array}$ \\
\hline
\end{tabular}


TABLE continued.

\begin{tabular}{|c|c|c|c|c|}
\hline Date. & $\begin{array}{l}\text { Thermo- } \\
\text { meter. }\end{array}$ & Time. & $\begin{array}{l}\text { Hygro. } \\
\text { meter. }\end{array}$ & Remarks. \\
\hline $\begin{array}{r}1827 . \\
\text { July } 16 . \\
17 . \\
\text { ", } \\
20 . \\
21 . \\
\text { ", }\end{array}$ & $\left.\begin{array}{l}65 \cdot 0 \\
64 \cdot 0 \\
84 \cdot 0 \\
67 \cdot 0 \\
71 \cdot 5 \\
78 \cdot 0\end{array}\right\}$ & $\begin{array}{l}12 \\
1 \text { p.m. } \\
\text { sun. } \\
2 \text { p.m. } \\
1 \text { p.m. } \\
\text { sun. }\end{array}$ & $\begin{array}{l}71 \cdot 0 \\
82 \cdot 5 \\
77 \cdot 0 \\
97 \cdot 1\end{array}$ & $\begin{array}{l}\text { Fair and windy. } \\
\text { Id. } \\
\text { Cloudy. } \\
\text { Clear and windy. }\end{array}$ \\
\hline \multicolumn{3}{|c|}{$\begin{array}{l}9 \text { days } 64^{\circ} \cdot 81 \text { max. } \\
53 \cdot 66 \text { min. }\end{array}$} & $59^{\circ} \cdot 2$ & Var. $50^{\circ}$ \\
\hline
\end{tabular}

Mountain of Cayambe, under the Equator.

\begin{tabular}{|c|c|c|c|c|}
\hline$" 24$. & 67.0 & 6 a.m. & 16.5 & $\begin{array}{c}\text { Elevation 12,705 feet. B. W. } \\
191^{\circ} \text {. Fair. } \\
\text { Elevation 14,217 feet. B. W. } \\
\text { 1881 foot,of the Nevado. } \\
\text { Weather fair. }\end{array}$ \\
\hline
\end{tabular}

Farm of Antisana.

\begin{tabular}{|c|c|c|c|c|}
\hline $\begin{array}{l}1899 . \\
\text { July } 1 .\end{array}$ & $\left.\begin{array}{l}33 \cdot 0 \\
35 \cdot 0 \\
38 \cdot 0 \\
45 \cdot 0 \\
67 \cdot 0 \\
43 \cdot 0 \\
37 \cdot 5\end{array}\right\}$ & $\begin{array}{l}6 \text { a.m. } \\
7 \\
8 \frac{1}{2} \\
2 \frac{1}{2} \text { p.m. } \\
\text { reflected } \\
4 \\
5 \frac{1}{2}\end{array}$ & $\begin{array}{r}30 \cdot 3 \\
\text { heat. }\end{array}$ & $\begin{array}{l}14,520 \text { feet. B. W. } 188^{\circ} \text {. } \\
\text { Fair and windy. } \\
\text { Bright. } \\
\text { Drifts of mists or Parametos. }\end{array}$ \\
\hline \multicolumn{5}{|c|}{$30^{\circ} \cdot 3$ Hygrometer. } \\
\hline
\end{tabular}

Descent from the Table-land of Quito to Esmeraldas, and Coast of the Pacific.

\begin{tabular}{|c|c|c|c|c|c|}
\hline Date. & $\begin{array}{c}\text { Thermo- } \\
\text { meter. }\end{array}$ & Time. & Hygrom. & Elevation, & Remarks. \\
\hline $\begin{array}{r}1828 . \\
\text { May } 14 . \\
15 . \\
17 .\end{array}$ & $\begin{array}{l}53 \cdot 0 \\
83 \cdot 0 \\
64 \cdot 0 \\
65 \cdot 5\end{array}$ & $\begin{array}{l}\}^{12} \text { sun. } \\
3 \text { p.m. } \\
\text { mean of } \\
10 \text { obs. }\end{array}$ & $\begin{array}{c}0 \\
30 \cdot 3\end{array}$ & $\begin{array}{c}12,986 \mathrm{ft} . \\
\text { B.W. } 190^{\circ} \frac{1}{2} \\
8772 \text { feet. } \\
\text { B.W. } 197 \frac{1}{2}^{\circ} \\
3932 \\
\text { B.W. } 205 \frac{1}{2}^{\circ}\end{array}$ & $\begin{array}{l}\text { Height of Pichan. } \\
\text { Descent of Pugsi. } \\
\text { Mindo. }\end{array}$ \\
\hline
\end{tabular}

Phil. Mag. S. 3. Vol. 15. Supplement. No.99. 1839. 2 L 
514 Col. R. Wright's Meteorological Observations made

Descent from Table-land.

TaBLE continued.

\begin{tabular}{|c|c|c|c|c|c|}
\hline Date. & $\begin{array}{l}\text { Thermo- } \\
\text { meter. }\end{array}$ & Time. & Hygrom. & Elevation. & Remarks. \\
\hline 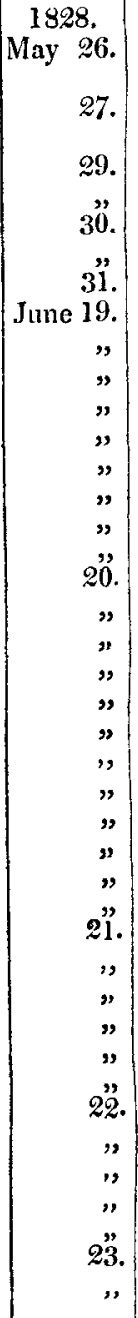 & $\begin{array}{c}0 \\
\cdots \\
70 \cdot 0 \\
72 \cdot 0 \\
78 \cdot 0 \\
72 \cdot 0 \\
74 \cdot 0 \\
74 \cdot 0 \\
73 \cdot 0 \\
73 \cdot 0 \\
75 \cdot 0 \\
77 \cdot 0 \\
79 \cdot 0 \\
76 \cdot 0 \\
80 \cdot 0 \\
82 \cdot 0 \\
76 \cdot 0 \\
73 \cdot 0 \\
73 \cdot 0 \\
72 \cdot 5 \\
74 \cdot 0 \\
74 \cdot 0 \\
76 \cdot 0 \\
78 \cdot 0 \\
82 \cdot 0 \\
77 \cdot 0 \\
83 \cdot 0 \\
80 \cdot 0 \\
76 \cdot 0 \\
72 \cdot 0 \\
77 \cdot 0 \\
77 \cdot \frac{3}{2} \\
79 \cdot 0 \\
78 \cdot 5 \\
73 \cdot 0 \\
70 \cdot 0 \\
74 \cdot 0 \\
78 \cdot 0 \\
76 \cdot 5 \\
79 \cdot 5 \\
73 \cdot 0 \\
80 \cdot 5\end{array}$ & 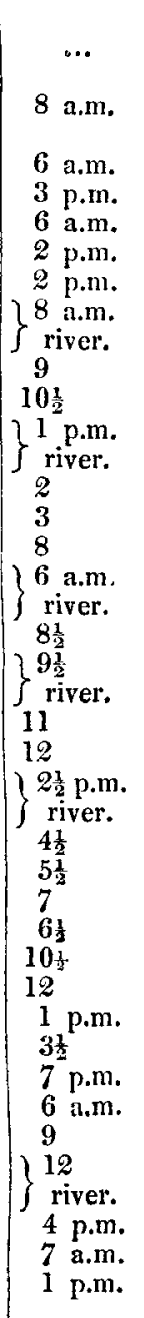 & \begin{tabular}{|c|}
$22 \cdot 0$ \\
bright. \\
$33 \cdot 0$ \\
$11 \cdot 0$ \\
cloudly. \\
misty \& \\
wind. \\
showery. \\
fair.
\end{tabular} & 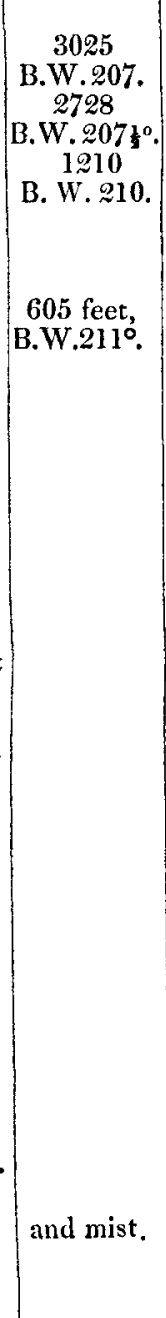 & $\begin{array}{l}\text { Canigne, abont } 12 \\
\text { leagues from the } \\
\text { coast, surrounded } \\
\text { by deep forests. }\end{array}$ \\
\hline & Thern & etric & $10^{\circ} 50^{\circ}$ & $\begin{array}{l}\text { 33. Hygro } \\
\text {. Hygrom }\end{array}$ & $\begin{array}{l}\text { ter } 22^{\circ} \cdot 77 \\
\text { rical do. } 22^{\circ} \text {. }\end{array}$ \\
\hline
\end{tabular}


in Colombia between the Weana-1880 and 1890.

Observations at Canigue, by Col. Brooke Young.

\begin{tabular}{|c|c|c|c|c|c|c|}
\hline 1828. & $6 \mathrm{a}, \mathrm{m}$ & Noon. & 3 p.m. & 6 p.m. & Medium. & Weather. \\
\hline $\begin{aligned} \text { Jan. } 19 . \\
20 . \\
21 . \\
\text { Feb. } 2 . \\
3 . \\
4 . \\
5 . \\
6 . \\
7 . \\
8 . \\
9 . \\
10 . \\
11 . \\
12 . \\
13 . \\
14 . \\
15 . \\
16 . \\
17 . \\
18 . \\
19 . \\
20 . \\
21 . \\
22 .\end{aligned}$ & $\begin{array}{c}0 \\
74 \cdot 0 \\
73 \cdot 0 \\
72 \cdot 0 \\
72 \cdot 0 \\
71 \cdot 0 \\
75 \cdot 0 \\
73 \cdot 0 \\
72 \cdot 0 \\
73 \cdot 0 \\
73 \cdot 5 \\
73 \cdot 0 \\
73 \cdot 0 \\
74 \cdot 0 \\
73 \cdot 0 \\
73 \cdot 5 \\
74 \cdot 0 \\
75 \cdot 0 \\
74 \cdot 0 \\
73 \cdot 0 \\
75 \cdot 0 \\
75 \cdot 0 \\
73 \cdot 0 \\
73 \cdot 0 \\
72 \cdot 0\end{array}$ & $\begin{array}{c}0 \\
82 \cdot 5 \\
81 \cdot 0 \\
85 \cdot 0 \\
85 \cdot 0 \\
86 \cdot 0 \\
78 \cdot 0 \\
81 \cdot 0 \\
83 \cdot 0 \\
79 \cdot 5 \\
83 \cdot 0 \\
86 \cdot 0 \\
83 \cdot 0 \\
80 \cdot 5 \\
83 \cdot 0 \\
86 \cdot 0 \\
82 \cdot 0 \\
83 \cdot 0 \\
79 \cdot 0 \\
80 \cdot 0 \\
84 \cdot 0 \\
85 \cdot 0 \\
83 \cdot 0 \\
84 \cdot 0 \\
80 \cdot 0\end{array}$ & $\begin{array}{c}0 \\
82 \cdot 0 \\
79 \cdot 0 \\
83 \cdot 0 \\
84 \cdot 0 \\
90 \cdot 0 \\
78 \cdot 0 \\
84 \cdot 0 \\
86 \cdot 0 \\
80 \cdot 0 \\
84 \cdot 0 \\
86 \cdot 0 \\
87 \cdot 0 \\
85 \cdot 0 \\
85 \cdot 0 \\
860 \\
83 \cdot 0 \\
86 \cdot 5 \\
79 \cdot 0 \\
87 \cdot 0 \\
89 \cdot 0 \\
88 \cdot 0 \\
82 \cdot 0 \\
89 \cdot 0 \\
85 \cdot 0\end{array}$ & $\begin{array}{c}0 \\
76.0 \\
75 \cdot 0 \\
77.0 \\
76 \cdot 0 \\
81.0 \\
760 \\
77.0 \\
78.0 \\
77 \cdot 5 \\
78.5 \\
77.0 \\
80.0 \\
77.0 \\
81.0 \\
77 \cdot 5 \\
77 \cdot 0 \\
79 \cdot 0 \\
76.0 \\
79.0 \\
79 \cdot 0 \\
79 \cdot 0 \\
79.0 \\
81.0 \\
81.0\end{array}$ & 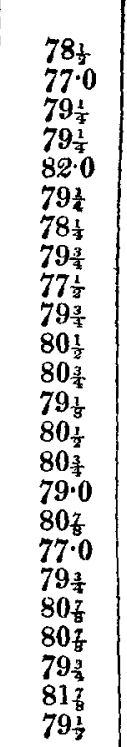 & $\begin{array}{l}\text { Continual rain. } \\
\text { Heavy showers. } \\
\text { Light showers. } \\
\text { Heavy showers. } \\
\text { Fair. } \\
\text { Continual rain. } \\
\text { Fair until } 5 \text { p.m. } \\
\text { Do. } \\
\text { Heavy rain. } \\
\text { Light showers. } \\
\text { Rain after } 5 \text { p.m. } \\
\text { Fair. } \\
\text { Rain until } 10 \text { a.m. } \\
\text { Do. } \\
\text { Showers after } 3 \text { p.m. } \\
\text { Showery. } \\
\text { Rain after } 6 \text { p.m. } \\
\text { Heavy showers. } \\
\text { Showers after } 4 \text { p.m. } \\
\text { Fair. } \\
\text { Id. } \\
\text { Showers after } 2 \text { p.m. } \\
\text { Rain after } 6 \text { p.m. } \\
\text { Rain all night. }\end{array}$ \\
\hline & \multicolumn{6}{|c|}{$\begin{array}{l}\text { Medium of the rainy season } 79^{\circ} 0 \text { nearly, } \\
\text { of the dry season } 74_{0} 56 \text {. } \\
\text { of the year...... } 76 \cdot 78 \text {. }\end{array}$} \\
\hline
\end{tabular}

River of Esmeraldas.

\begin{tabular}{|c|c|c|c|c|c|}
\hline 1828. & Air. & Water. & Hygrom. & Time. & Weather. \\
\hline $\begin{array}{r}\text { June } 12 . \\
13 . \\
13 \\
14 . \\
13 \\
16 . \\
18 .\end{array}$ & $\begin{array}{l}76.0 \\
78 \cdot 0 \\
76 \cdot 0 \\
79 \cdot 0 \\
79 \cdot 5 \\
75 \cdot 0 \\
80.5 \\
80.0 \\
80.5 \\
73 \cdot 0\end{array}$ & $\begin{array}{l}75 \cdot 5 \\
77 \cdot 5 \\
75 \cdot 0 \\
76 \cdot 0 \\
76 \cdot 0 \\
74 \cdot 5 \\
75 \cdot 0 \\
76 \cdot 0 \\
74 \cdot 5 \\
73 \cdot 0\end{array}$ & $\begin{array}{r}0 \\
5 \cdot 5 \\
0 \cdot 0 \\
0 \cdot 0 \\
5 \cdot 5 \\
8 \cdot 2 \\
0 \cdot 0 \\
11 \cdot 0 \\
13 \cdot 7 \\
13 \cdot 7 \\
0.0\end{array}$ & $\begin{array}{c}6 \frac{1}{2} \text { a.m. } \\
3 \frac{1}{2} \text { p.m. } \\
6 \text { a.m. } \\
10+ \\
3 \text { p.m. } \\
6 \frac{1}{3} \text { a.m. } \\
10 \\
12 \\
10 \text { a.m. } \\
6 \frac{1}{2} \text { a.m. }\end{array}$ & $\begin{array}{l}\text { Misty rain. } \\
\text { Do. } \\
\text { Do. } \\
\text { Cloudy. } \\
\text { Do. } \\
\text { Misty. } \\
\text { Fair. } \\
\text { Id. } \\
\text { Id. } \\
\text { Misty. }\end{array}$ \\
\hline Med. & $77 \cdot 75$ & $75 \cdot 4$ & $5 \cdot 76$ & & \\
\hline
\end{tabular}

$2 \mathrm{~L} 2$ 
516 Col. Wright's Meteorological Observations in Colombia.

Toron of Esmeraldas. Lat. $55^{\prime} \mathrm{N}$.

\begin{tabular}{|c|c|c|c|c|c|}
\hline 1828. & Therm. & & Hygrom. & Time. & Weather. \\
\hline 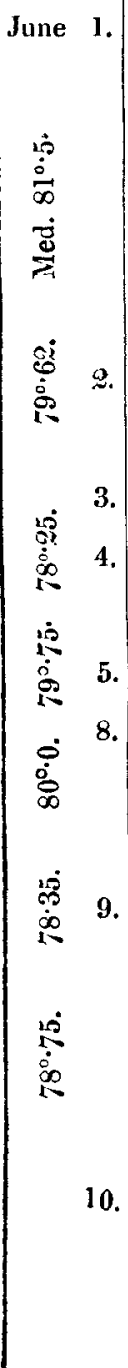 & $\begin{array}{l}76 \cdot 0 \\
\\
77 \cdot 0 \\
80 \cdot 0 \\
82 \cdot 0 \\
85 \cdot 0 \\
87 \cdot 0 \\
84 \cdot 0 \\
82 \cdot 0 \\
82 \cdot 0 \\
80 \cdot 0 \\
76 \cdot 0 \\
78 \cdot 0 \\
82 \cdot 5 \\
82 \cdot 0 \\
760 \\
80 \cdot 5 \\
76 \cdot 0 \\
80 \cdot 0 \\
85 \cdot 0 \\
780 \\
84 \cdot 0 \\
760 \\
75 \cdot 0 \\
78 \cdot 0 \\
83 \cdot 0 \\
84 \cdot 0 \\
82 \cdot 0 \\
76 \cdot 5 \\
75 \cdot 0 \\
760 \\
78 \cdot 0 \\
81 \cdot 0 \\
830 \\
82 \cdot 0 \\
80 \cdot 3 \\
78 \cdot 0 \\
78 \cdot 0 \\
78 \cdot 0 \\
77 \cdot 0 \\
76 \cdot 0 \\
80 \cdot 5 \\
83 \cdot 0 \\
83 \cdot 0 \\
80 \cdot 5\end{array}$ & Sun $84^{\circ}$. & $\begin{array}{l}19 \cdot 2 \\
16 \cdot 7\end{array}$ & 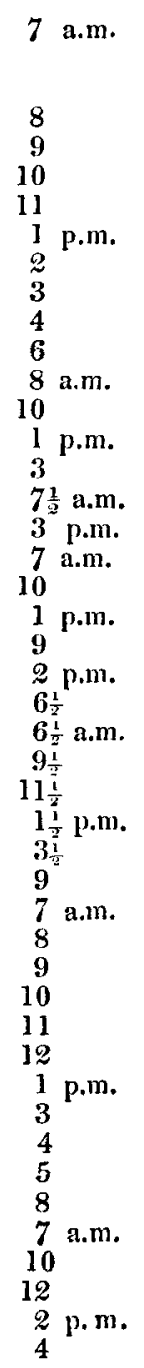 & $\begin{array}{l}\text { About six miles } \\
\text { from the sea- } \\
\text { coast. Elev. 0. } \\
\text { Cloudy. } \\
\text { Do. } \\
\text { Do. } \\
\text { Do. } \\
\text { Do. } \\
\text { Do. } \\
\text { Rain. } \\
\text { Do. } \\
\text { Do. } \\
\text { Cloudy. } \\
\text { Do.; rain. } \\
\text { Do. } \\
\text { Misty. } \\
\text { Do. } \\
\text { Do. } \\
\text { Fair. } \\
\text { Do. } \\
\text { Do. } \\
\text { Do. } \\
\text { Do. } \\
\text { Do. } \\
\text { Misty. } \\
\text { Fair. } \\
\text { Do. } \\
\text { Do. } \\
\text { Do. } \\
\text { Do. } \\
\text { Do. } \\
\text { Do. } \\
\text { Do. } \\
\text { Do. } \\
\text { Do. } \\
\text { Cloudy. } \\
\text { Rain. } \\
\text { Heavy rain. } \\
\text { Do. } \\
\text { Do. } \\
\text { Fair. } \\
\text { Do. } \\
\text { Do. } \\
\text { Cloudy. } \\
\text { Do. } \\
\text { Fair. } \\
\text { Dai. }\end{array}$ \\
\hline \multicolumn{6}{|c|}{$\begin{array}{l}\text { Thermometrical Var. } 10^{\circ} \text {. } \\
\text { Hygrometrical do. } 15 \cdot 6 .\end{array}$} \\
\hline
\end{tabular}


Sea-coast South of the River of Esmeraldas.

\begin{tabular}{|c|c|c|c|c|}
\hline 1827 & Therm. & Hygrom. & Time. & Weather. \\
\hline $\begin{array}{r}\text { May } 20 . \\
\\
21 . \\
\text { Dec. 1.5. } \\
16 . \\
18 . \\
19 .\end{array}$ & $\begin{array}{l}75 \cdot 0 \\
78 \cdot 0 \\
75 \cdot 0 \\
77 \cdot 5 \\
76 \cdot 4 \\
72 \cdot 6 \\
81 \cdot 5 \\
79 \cdot 0 \\
75 \cdot 5 \\
80 \cdot 0\end{array}$ & $\begin{array}{r}22 \cdot 0 \\
6.5 \\
19 \cdot 0 \\
20 \cdot 0 \\
5.5 \\
30 \cdot 0\end{array}$ & $\begin{aligned} 7 & \text { a.m. } \\
4 & \text { p.m. } \\
6 & \text { a.m. } \\
12 & \\
8 & \text { p.m. } \\
6 & \text { a.m. } \\
2 & \text { p.m. } \\
3 & \text { p.m. } \\
6 & \text { a.m. } \\
2 & \text { p.m. }\end{aligned}$ & $\begin{array}{l}\text { Atacames. Lat. } \\
52^{\prime} \mathrm{N} \text {. Cloudy. } \\
\text { Cloudy. } \\
\text { Cloudy. } \\
\text { Rain. } \\
\text { Cloudy. } \\
\text { Mompiche. } \\
\text { Clondy; rain at } \\
\text { night. } \\
\text { Clear. }\end{array}$ \\
\hline $\begin{array}{r}1830 . \\
\text { Nov. } 7 . \\
8 \\
9,10 . \\
11 . \\
12 . \\
13 .\end{array}$ & $\begin{array}{l}72 \cdot 0 \\
76 \cdot 0 \\
72 \cdot 0 \\
79 \cdot 0 \\
72 \cdot 0 \\
76 \cdot 0 \\
79 \cdot 0 \\
77 \cdot 0 \\
71 \cdot 0 \\
75 \cdot 0 \\
76 \cdot 28\end{array}$ & $22 \cdot 0$ & 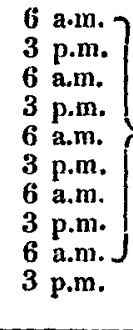 & $\begin{array}{l}\text { Wind, cloudy and } \\
\text { mists. } \\
\text { Rain. }\end{array}$ \\
\hline
\end{tabular}

LXXVII. Proceedings of Learned Societies.

ROYAL SOCI ETY.

Address of the Marquis of Northampton, President, read at the Anniversary Meeting, November 30, 1839.

Gentlemen,

A YEAR having now elapsed since you conferred upon me the

highly honourable office of your President, it becomes my duty, in accordance with the example of my predecessors, to address you. The first and most agreeable part of my task is to express my feelings of gratitude to those Gentlemen whom you were pleased to select as my Council. I am most highly indebted to them for the zealous co-operation and hearty assistance which $I$ have ever received at their hands. It is to them that $I$ have looked to aid my inexperience, and to supply my manifold deficiencies, and I have not been disappointed. To those who are the more permanent officers of the Society, the Treasurer and the Secretaries, my obligations are particularly great, and I will venture to add, that to them, as well as to the other members of the Council, your thanks are due as well as mine.

The past year has indeed been to that portion of the Royal So- 